\title{
ERRATUM
}

S. Kurth · D. Bulian · B. Kreft · Th. Riemenschneider

\section{Intraarterial hepatic chemotherapy with fluorouracil, fluorodeoxyuridine, mitomycin C, cisplatin or methotrexate as single-agent anticancer drugs for a transplanted experimental liver tumor in rats}

In: J Cancer Res Clin Oncol (1996) 122: 421-426

The values given in the row "Mean tumor volume day 12 $\left(\mathrm{mm}^{3}\right)$ " were actually total volumes for each group. The correct mean values are those given below:

Table 1 Main values of parameters measured ( $n$ number of cases). The mean tumor multiplication factor $(T M F)$ is calculated from all single TMF values of a group

\begin{tabular}{|c|c|c|c|c|c|c|}
\hline Parameter & Control & FdUrd & FUra & Mitomycin C & Cisplatin & Methotrexate \\
\hline$n$ & 13 & 12 & 14 & 13 & 11 & 12 \\
\hline Dose $(\mathrm{mg} / \mathrm{kg})$ & - & 400.0 & 90.0 & 6.0 & 7.0 & 16.0 \\
\hline $\begin{array}{l}\text { Mean tumor volume } \\
\text { day } 5\left(\mathrm{~mm}^{3}\right)\end{array}$ & 154.4 & 126.2 & 157.6 & 176.1 & 136.8 & 136 \\
\hline $\begin{array}{l}\text { Mean tumor volume } \\
\text { day } 12\left(\mathrm{~mm}^{3}\right)\end{array}$ & 1440.0 & 409.5 & 587.7 & 143.8 & 90.6 & 23.1 \\
\hline $\begin{array}{l}\text { Animal weight days } \\
5-12\end{array}$ & -1 & -17 & -13 & -22 & -7 & -11 \\
\hline Mean TMF & 9.66 & 3.78 & 3.03 & 0.96 & 0.64 & 0.15 \\
\hline
\end{tabular}

S. Kurth $\cdot$ D. Bulian

Klinik und Poliklinik fuir Chirurgie der Universität Bonn, (Direktor

Prof. Dr. med. A. Hirner) Sigmund Freud Str. 25, 53105 Bonn,

Germany

B. Kreft

Radiologische Klinik der Universität Bonn, (Direktor

Prof. Dr. med. H. Schild) Sigmund Freud Str. 25, 53105 Bonn,

Germany

Th. Riemenschneider (

DRK-Krankenhaus Neuwied, 56504 Neuwied, Germany 